\title{
Effect of Mutual Inductance on Steady-state Performance and Position Estimation of Switched Reluctance Motor Drive
}

\author{
Debiprasad Panda, Student Member, IEEE and V. Ramanarayanan \\ Department of Electrical Engineering, \\ Indian Institute of Science, Bangalore 560012, India \\ email: dpanda@ee.iisc.ernet.in, vram@ee.iisc.ernet.in
}

\begin{abstract}
In this paper, a practical way of measuring the mutual flux in Switched Reluctance (SR) motor is explained and the test results for an $8 / 6$ pole, $4 \mathrm{~kW}$ motor are presented. The performance of the drive is studied in simulation with and without mutual flux. The effect of mutual flux on phase current, flux and average torque is demonstrated with simulation result. Further the effect of same on position estimation is verified in simulation and experiment. It is shown that appropriate correction for mutual flux may improve the accuracy of estimated position by $3^{\circ}$.
\end{abstract}

\section{INTRODUCTION}

The design, performance evaluation and high performance control of Switched Reluctance (SR) motor requires detailed knowledge of the flux-linkage characteristics of the windings for different rotor positions at different excitation currents. Most of the existing literature [1-2] employ flux-linkage characteristics obtained from static tests or through finite element method. Dynamic effects and mutual couplings between different phases are ignored. It is reported that mutual coupling can cause errors in the flux-linkage characteristics as much as $7 \%$ [3]. For accurate modelling the mutual coupling needs to be considered.

Traditionally SR motor needs position sensors for satisfactory control. Generally, absolute encoder or discrete optical and magnetic sensors are used for position sensing. These sensors inherently reduce the reliability of the drive. Extensive application of SR motor will require development of robust sensorless control. Such sensorless control schemes need accurate position estimation, which in turn needs accurate evaluation of flux-linkage characteristics accounting for the non-idealities of dynamic effects and mutual coupling.

This paper attempts to model the mutual coupling effects on the flux-linkage characteristics. After indicating the qualitative effects, the mutual coupling is experimentally determined. Measurement method, experimental set-up and the test results are presented. The effect of mutual coupling on the phase currents, phase fluxes and energy conversion contours are verified through simulation. The effect of the same on position estimation is verified through simulation and experiment. A more accurate position estimation algorithm is presented considering the mutual coupling. It is demonstrated that the accuracy of position estimation is considerably improved following the proposed strategy.

\section{Mutual Flux and Its Measurement}

The measurement of static flux-linkage characteristics are well documented in literature [4, 5]. In those measurements, excitation is given to only one phase while other phases are kept open. These off-line measured characteristics do not take into account the mutual fluxes due to the other phases. During dynamic operation more than one phase will have excitation currents at a time and the flux-linkages of each phase will be influenced by the currents in the other conducting phases. Hence, proper modelling and accurate analysis require the knowledge of mutual fluxes in addition to the flux linkage characteristics of the individual phases.

\section{A. Mutual coupling between neighbouring phases}

In the test motor, the winding connections are such that the direction of the fluxes (marked by arrows) in each of the individual phases are as shown in Fig. 1. The arrows in Fig. 1 indicate the direction of the individual phase fluxes. The figure suggests that the mutual flux will not be in the same direction for all phases. The mutual flux in $\mathrm{Ph} 2$ and $\mathrm{Ph} 3$ due to their neighbouring phases are additive. On the contrary, the same in $\mathrm{Ph} 1$ and $\mathrm{Ph} 4$ are in opposing nature. The typical mutual flux pattern due to $\mathrm{Ph} 1$ with its neighbouring phases (Ph4 and $\mathrm{Ph} 2$ ) are shown in Figs $2 \mathrm{a}, 2 \mathrm{~b}$ and $2 \mathrm{c}$. When $\mathrm{Ph} 1$ is at $0^{\circ}$ (Fig. 2a), the mutual linkage due to $\mathrm{Phl}$ with both its neighbouring phases are equal. At $22.5^{\circ}$ (Fig. 2b) the mutual coupling between $\mathrm{Ph} 1$ and $\mathrm{Ph} 4$ will be maximum as the air-gap between the stator and the rotor along the mutual flux

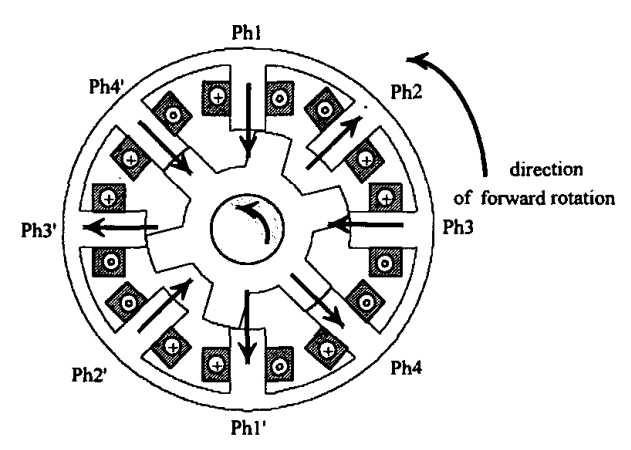

Fig. 1 Flux Pattern (flux due to an individual phase) at poles of a 4 phase $8 / 6$ SR Motor 
path is minimum at that position. Similarly, at $37.5^{\circ}$ (Fig. 2c) the mutual coupling between $\mathrm{Ph} 1$ and $\mathrm{Ph} 2$ is maximum. The mutual coupling at other angular positions will take intermediate values. An experiment is conducted to verify the nature and measure the magnitude of mutual fluxes of each phase. The details of the experiment is explained below.

\section{B. Measurement of mutual flux}

The rotor is held standstill at a predetermined position using an indexing head and a disk with position marking, is. mounted on the shaft of the motor for verifying the position of the rotor. The experimental set up is given in Fig. 3. One of the phases of the motor is connected in series with a rheostat (Rh) and an electronic switch (S) to a dc source, with all other phases open. Voltages are induced in the unenergised phases due to the rate of change of current in the active phase. The induced voltages in the unenergised phases and the current through the active phase are recorded and stored in a DSO (Digital Storage Oscilloscope). The rheostat ( $\mathrm{Rh}$ ) is used to limit the current to the required value. The experiment is conducted over a complete electric cycle $\left(60^{\circ}\right.$ mechanical) and the test results are stored for every $2^{\circ}$. The typical test results of current in the active phase and induced voltages in the other phases due to the main phase current are shown in Figs $4 \mathrm{a}-4 \mathrm{~d}$. From the plots, it can be seen that the induced voltage in the phase in quadrature $(\mathrm{Ph} 3)$ with the excited phase (Phl) is very much less compared to the adjacent phases ( $\mathrm{Ph} 2$ and $\mathrm{Ph} 4$ for this case). Hence, the mutual linkage due to the quadrature phase may be ignored. It can also be observed that the induced voltage waveforms in the two neighbouring phases of the active phase are similar both at $0^{\circ}$ and $30^{\circ}$ (c.f. Fig. $4 \mathrm{a}$ and $4 \mathrm{~b}$ ). The magnetic path in Fig. 2a justifies this phenomenon. The mutual linkage with $\mathrm{Ph} 4$ and $\mathrm{Ph} 2$ are maximum when $\mathrm{Ph} 1$ is at $22.5^{\circ}$ and $37.5^{\circ}$ respectively. These observations also can be justified by the Fig. 2b and Fig. 2c.

The mutual flux $\Psi_{j k}$ is computed through the numerical integration of the induced voltage $V_{j k}$ in the $\mathrm{j}$-th phase due to the excitation in the $\mathrm{k}$-th phase and that is given by the following equation.

$$
\Psi_{j k}=\int V_{j k} d t
$$

The typical plots of the computed mutual flux-linkages are shown over a complete electrical cycle in Fig. 5 and Fig. 6 for different excitation currents. In these plots, $x$-axis represents the relative position of the rotor pole with respect to the phase being excited and these positions are meant for forward rotation of the motor (the arrows in Fig. 1 and Fig. 2 indicate the forward rotation). The same experiment is repeated for different phases and each time the mutual flux to the neighbouring phases are computed.

\section{Discussion:}

The test results confirm that the magnitude of the mutual flux in the neighbouring phases are identical and follow the (a)

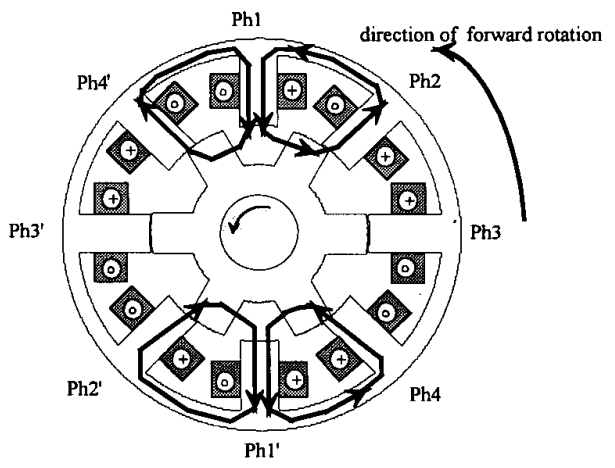

(b)

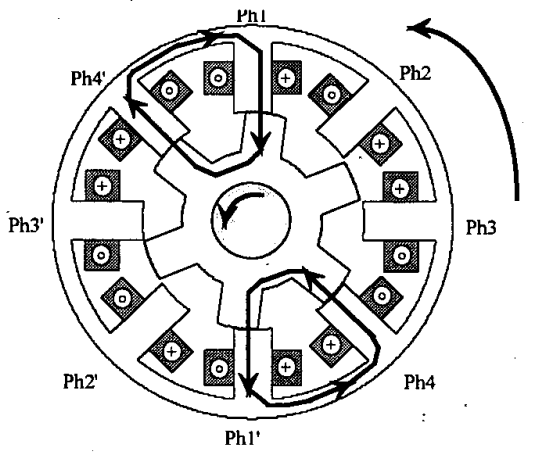

(c)

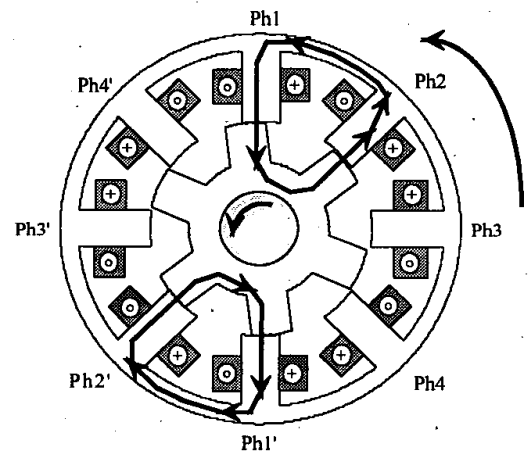

Fig. 2 Mutual Flux-linkages to its neighbouring phases when $\mathrm{Phl}$ is excited: (a) $\mathrm{Ph} 1$ is at $0^{\circ}$, (b) $\mathrm{Ph} 1$ at $22.5^{\circ}$, (c) $\mathrm{Ph} 1$ is at $37.5^{\circ}$

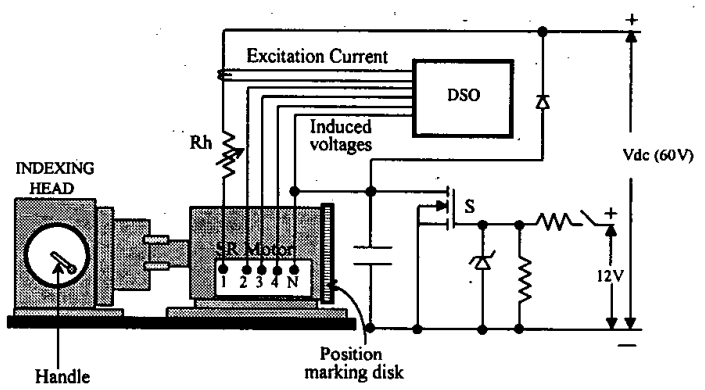

Fig. 3. Experimental set-up for Mutual flux-linkage measurement $(1,2,3,4$ represents the different phase and $\mathrm{N}$ is the neutral connection of the phase windings) 

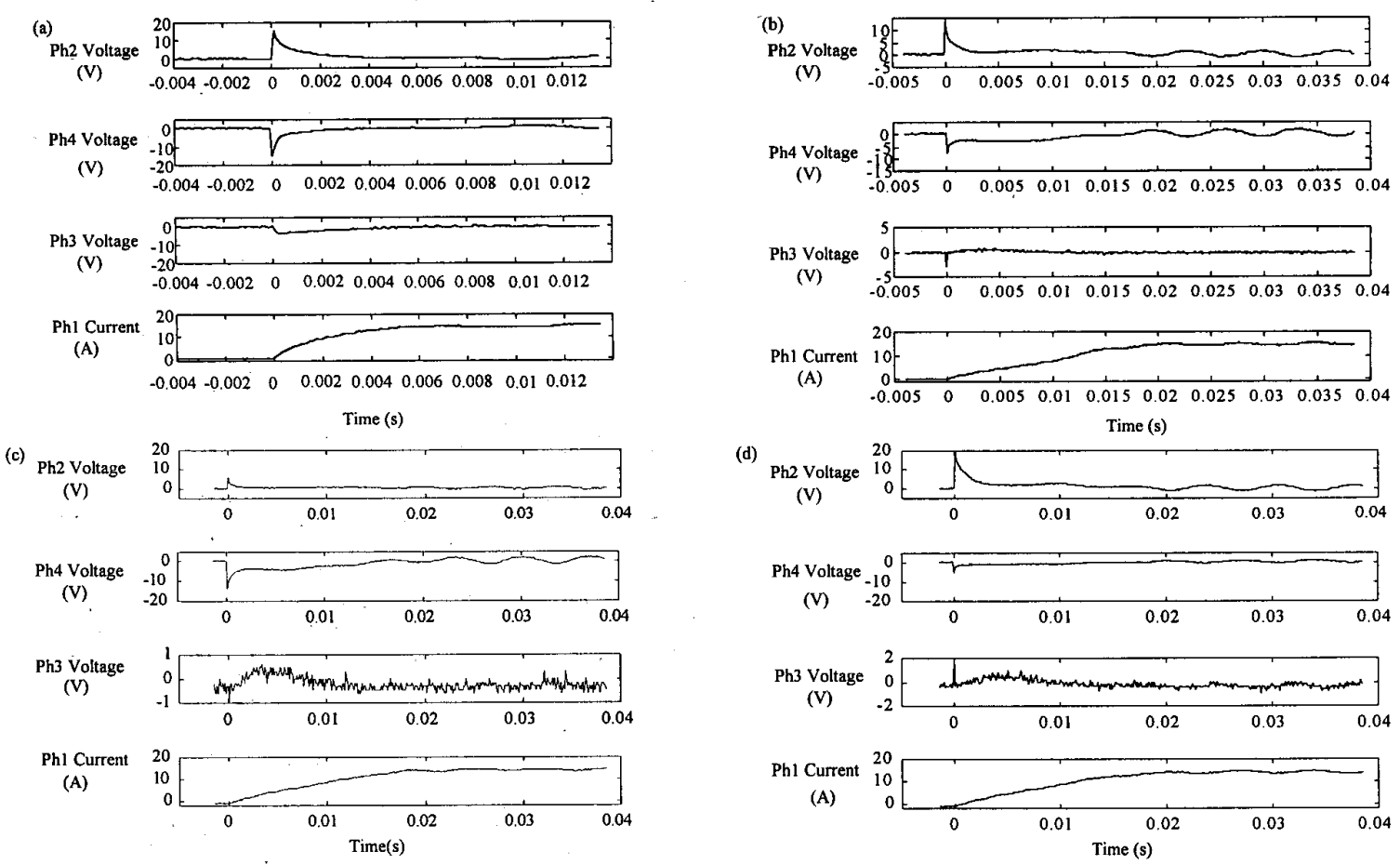

Fig. 4 Mutually induced voltages in different phases due to excitation in phasel at (a) position $0^{\circ}$, (b) position $30^{\circ}$, (c) position $22.5^{\circ}$ and (d) position $37.5^{\circ}$.

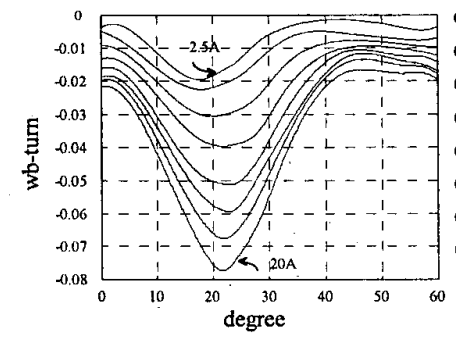

(i) Ph4 flux when Phl is excited

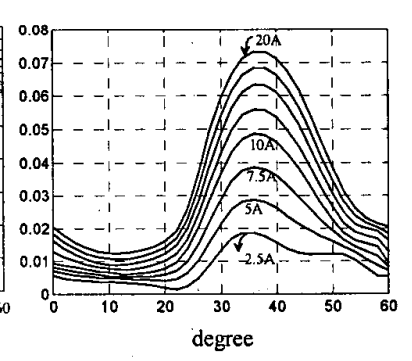

(ii) $\mathrm{Ph} 2$ flux when $\mathrm{Ph} 1$ is excited

Fig. 5. Experimentally obtained Mutual Flux -linkages in (i) $\mathrm{Ph} 4$ and (ii) $\mathrm{Ph} 2$ for the different excitation currents $(0: 2.5: 20 \mathrm{~A})$ in $\mathrm{Ph} 1$.

same pattern for all the phases. On the contrary, the direction of the mutual fluxes in different phases are not the same. The direction depends on the winding connection, which is a designer prerogative.

It may be seen in Figs 5 and 6 , the mutual flux at $22.5^{\circ}$ with the preceding phase (phase sequence is $\mathrm{Ph} 1-\mathrm{Ph} 2-\mathrm{Ph} 3-\mathrm{Ph} 4-\mathrm{Ph} 1$ ) is maximum. Similarly, at $37.5^{\circ}$ the mutual flux with the following phase is at maximum. This is in conformity with the earlier comments with respect to Figs $2 \mathrm{a}, 2 \mathrm{~b}$ and $2 \mathrm{c}$.

It is observed that the magnitude of peak mutual flux linkage is around $\pm 10 \%$ of the self-flux of an individual phase at rated current. The static flux-linkage characteristics of the test motor are given in Fig. 7. Analysis and

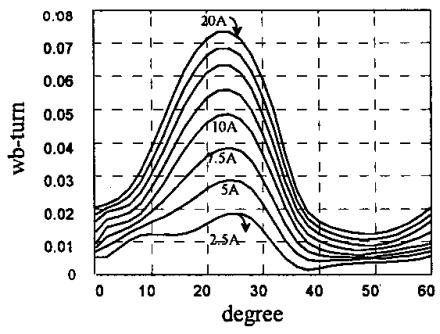

(i) $\mathrm{Ph} 2$ flux when $\mathrm{Ph} 3$ is excited

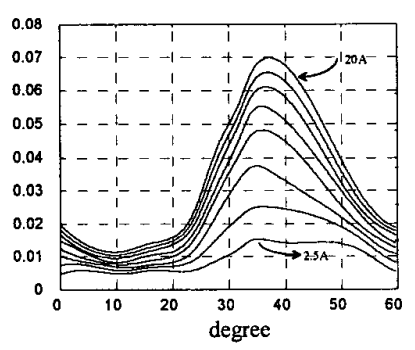

(ii) $\mathrm{Ph} 4$ flux when $\mathrm{Ph} 3$ is excited
Fig. 6. Experimentally obtained Mutual Flux -linkage in (i) $\mathrm{Ph} 2$ and (ii) $\mathrm{Ph} 4$ for the different excitation currents $(0: 2.5: 20 \mathrm{~A})$ in $\mathrm{Ph} 3$

quantification of mutual flux-linkage is useful for various reasons. Its influence in dynamic condition can be studied through simulation. In many position estimation algorithms of SR motor, the flux-linkage characteristics may be corrected for these mutual fluxes to obtain higher accuracy in estimation.

\section{Simulation with Mutual Flux}

The electromagnetic characteristics of the SR motor may be represented by the following equations-

$$
v_{j}=R_{j} i_{j}+\frac{d \Psi_{j}}{d t}
$$




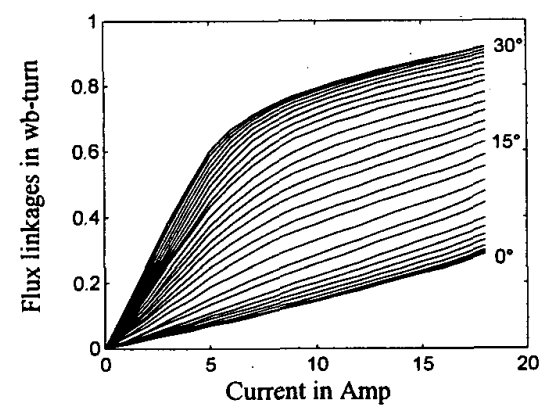

Fig. 7 Experimentally obtained static Flux-linkage characteristics for different positions $\left(0^{\circ}: 1^{\circ}: 30^{\circ}\right)$ and currents $(0: 1: 18 \mathrm{~A})$

$$
\begin{aligned}
& \Psi_{j}=\Psi_{j j}+\sum_{j=1} \Psi_{j k, 2,3,4} \\
& \Psi_{j k}=f\left(i_{k}, \theta_{k}\right) \\
& W_{j}^{\prime}=\int \Psi_{j} d i_{j} \\
& T a v_{j}=\frac{\Delta W_{j}^{\prime}}{\Delta \theta_{j}}
\end{aligned}
$$

where $v_{j}, R_{j}, i_{j}, \Psi_{j}, \Psi_{j j}, \Psi_{j k}, \theta_{j} \quad$ represent the voltage, resistance, current, net flux-linkage, self flux-linkage, mutual flux-linkage and relative position of the active phase ( $j$-th phase). The current and position of the other conducting phases (k-th phase) are represented as $i_{k}$ and $\theta_{k}$ respectively; the average torque, co-energy and conduction angle of the active phase are represented as $T a v_{j}, W_{j}^{\prime}$ and $\Delta \theta_{j}$ respectively. Average torque due to a particular phase over a complete phase cycle is computed from the change in co-energy during that cycle using (6).

Measured mutual flux-linkage and static flux-linkage characteristics as given in Figs. 5, 6 and 7 are stored in the form of look up tables. The well-known time step integration method is used for simulation [6]. The following assumptions are made -

1. eddy current effects are neglected

2. dc bus voltage is assumed to be ripple free.

The phase voltage is a function of the dc supply voltage and the states of the switches in the controller. At any instant, the phase flux linkage is obtained by integrating (2) over the previous time step. Now with a known position $\theta_{j}$ and known flux $\Psi_{j}$, the value of $i_{j}$ may be found using pre-stored static flux-linkage characteristics $\left(i_{j}=f\left(\theta_{j}, \Psi_{j j}\right)\right)$. In this process mutual flux is ignored.

While mutual fluxes are considered, the flux obtained through (2) should be corrected for the mutual flux due to the other conducting phases. This corrected flux is the self flux

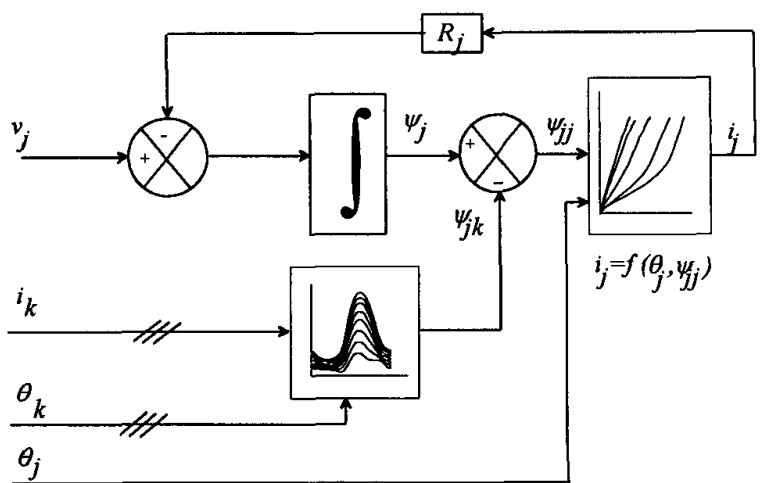

Fig. 8 Simulation block diagram of the electrical subsystem of SR motor with flux-linkage characteristics in the form of a look-up table

$\left(\Psi_{i j}\right)$. Knowing self flux and position, the corresponding current may be obtained following the same process as mentioned above. The detailed simulation block diagram is given in Fig. 8.

The conventional control method employs two regimes of control namely low speed chopping control and high speed pulse width control [5]. In order to illustrate the effect of mutual coupling throughout the speed range, two case studiesone at very low speed $(75 \mathrm{rpm})$ and another at rated speed $(1500 \mathrm{rpm})$ - are presented here.

\section{A. casel}

Simulation is carried out at very low speed (75 rpm) with T-on ( position at which phases start conducting) and T-off (position at which phases are switched off) angles at $0^{\circ}$ and $30^{\circ}$ respectively; current reference $I^{*}$ at $18 \mathrm{~A}$ and dc excitation voltage at 280 volt. The switching frequency of the converter is assumed to be large ( $200 \mathrm{kHz}$ in this case). The simulation is conducted first assuming the mutual fluxes to be zero. The net fluxes and energy conversion loops in different phases are shown in Fig. 9a and Fig. 9b respectively. It shows that without mutual fluxes, the fluxes and energy conversion loops in all the phases are equal. It is well known that the average torque of any phase is proportional to the area covered by its energy conversion loop [5] in one phase cycle. The simulation results of phase current, net flux and mutual flux waveforms in the different phases with the mutual fluxes are given in Figs. $10 \mathrm{a}$ and $10 \mathrm{~b}$. It can be seen that the flux patterns are not the same for all phases. The flux waveforms for $\mathrm{Ph} 2$ and $\mathrm{Ph} 3$ are similar, whereas they are different for $\mathrm{Ph} 4$ and $\mathrm{Ph} 1$. The peak flux of $\mathrm{Ph} 4$ is less compared to all other phases. The self flux and net flux for the different phases are compared in Fig. 11 (i) to 11 (iv). The results show that the net fluxes for all the phases are not the same. Hence, the flux-current contour for each of the phases are expected to follow different trajectories. Hence the average torque produced in all the phases will not be identical. At $75 \mathrm{rpm}$ and full load, the energy conversion loops of all the phases are shown in Fig. 12 with and without considering mutual fluxes. Fig. 13 gives the 
(a)

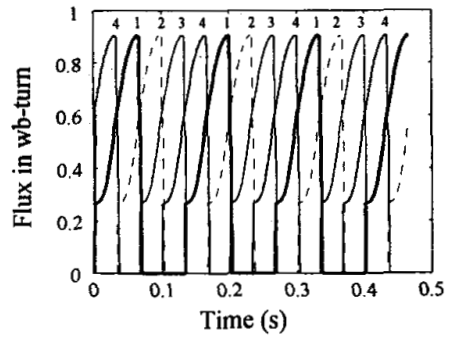

(b)

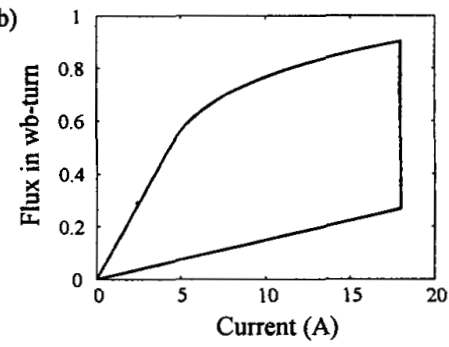

Fig. 9 Simulation results without mutual flux at $75 \mathrm{rpm}$ and full load: (a) All four phase fluxes; (b) Energy conversion loops of all phases; 1,2,3,4 represent $\mathrm{Ph} 1, \mathrm{Ph} 2, \mathrm{Ph} 3$ and $\mathrm{Ph} 4$ respectively flux), $\mathrm{T}$-on $=0^{\circ}$, $\mathrm{T}$-off $=30^{\circ}$.

(a) (i)

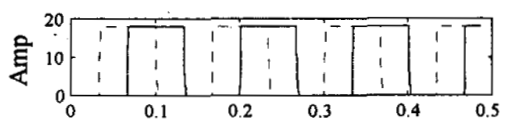

(ii)

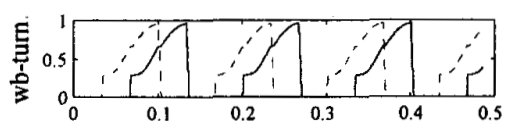

(iii)

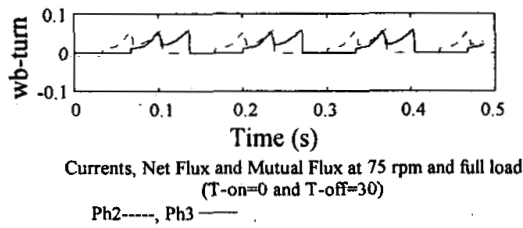

(b)

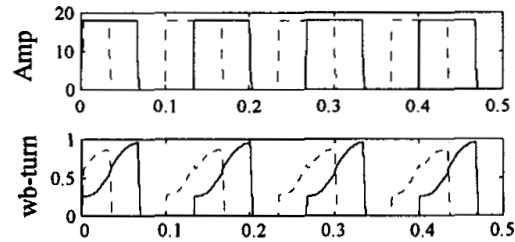

(iii)

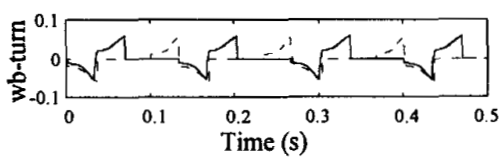

Currents, Net Flux and Mutual Flux at 75 rpm and full load ( $T$-on $=0$ and $T$-off $=30$ )

Fig. 10 Simulation results at $75 \mathrm{rpm}$ and full load: (i) current, (ii) Net flux, (iii) Mutual flux for (a) $\mathrm{Ph} 2$ and $\mathrm{Ph} 3$; and (b) Phland $\mathrm{Ph} 4$

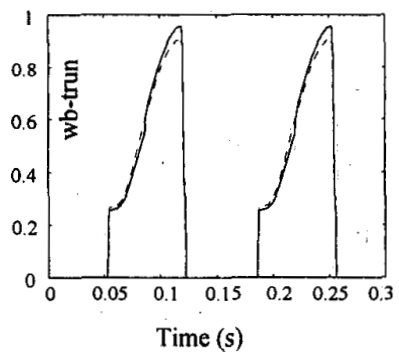

(i)

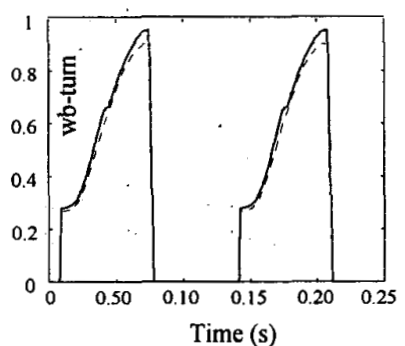

(ii)

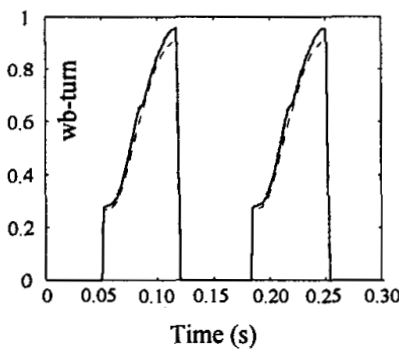

(iii)

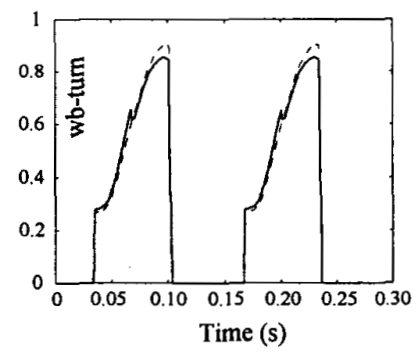

(iv)

Fig. 11 Comparison of net flux (bold line) and self flux (dotted line) at $75 \mathrm{rpm}$ and full load for: (i) Ph1, (ii) Ph2, (iii) Ph3 and (iv) Ph4 (Simulation results considering the mutual flux). $\left(\mathrm{T}-\mathrm{on}=0^{\circ}, \mathrm{T}\right.$-off $\left.=30^{\circ}\right)$
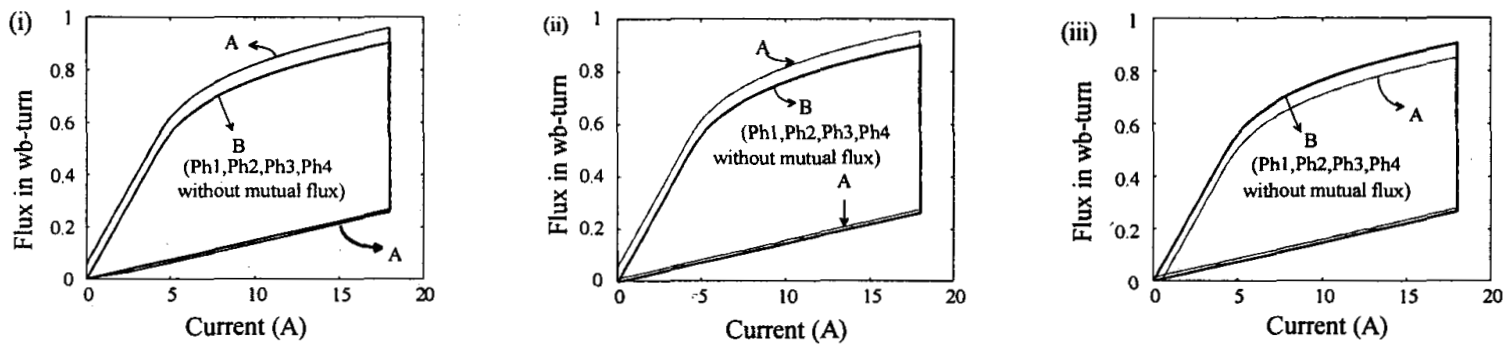

Fig. 12 Comparison of energy conversion loops of (i) $\mathrm{Ph} 1$ (ii) $\mathrm{Ph} 2$ and $\mathrm{Ph} 3$ (iii) $\mathrm{Ph} 4$ : (A) with and; (B) without mutual fluxes at $75 \mathrm{rpm}$ and full load .

comparison of energy conversion loops among different phases when mutual flux is considered. The results with mutual flux show that the average torque produced in $\mathrm{Ph} 1$ will be maximum and that in $\mathrm{Ph} 4$ will be minimum. The average torque in $\mathrm{Ph} 2$ and $\mathrm{Ph} 3$ are equal but they are less than that in $\mathrm{Ph} 1$ and more than in $\mathrm{Ph} 4$. Hence, it can be concluded that for a fixed current operation and fixed $\mathrm{T}$-on and $\mathrm{T}$-off angles, the load sharing varies in different phases due to 

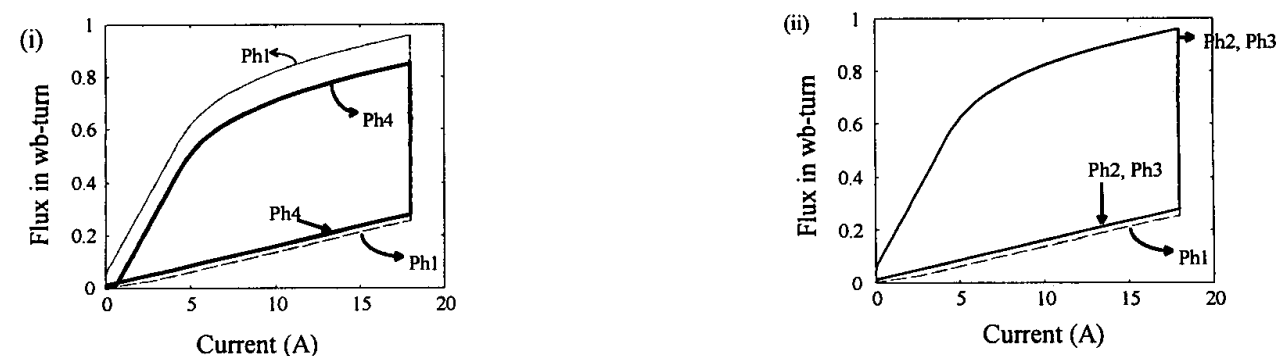

Fig. 13 Comparison of energy conversion loops with mutual fluxes (i) between $\mathrm{Ph} 1$ and $\mathrm{Ph} 4$ and (ii) between $\mathrm{Ph} 1$ and $\mathrm{Ph} 2$ (Ph3) at 75 rpm and full load .
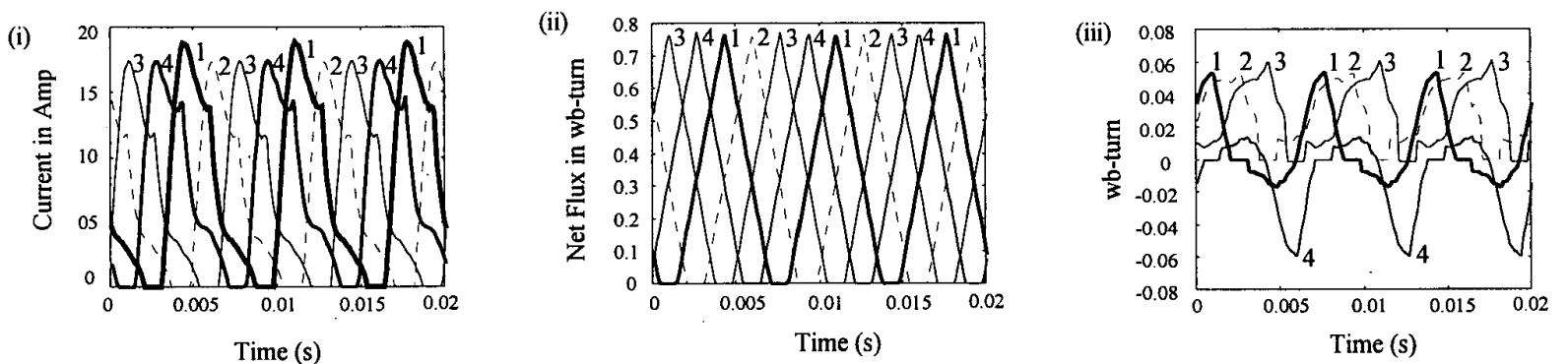

Fig. 14 Simulation results at 1500 ipm and full load: (i) currents, (ii) net fluxes and (iii) mutual flux-linkages for different phases. 1-2-3-4 represents the $\mathrm{Ph} 1, \mathrm{Ph} 2, \mathrm{Ph} 3$ and $\mathrm{Ph} 4$ respectively.

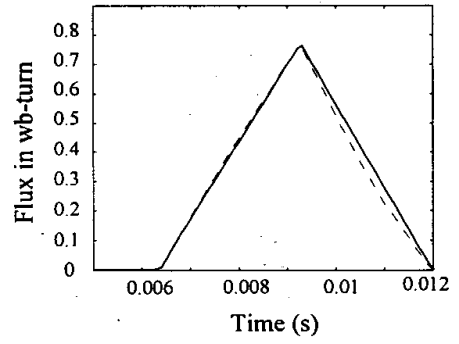

Fig. 15 Comparison of net flux (solid) and self flux (dotted) for Phl at 1500 rpm and full load when mutual flux is considered.

mutual inductance effect. This may also introduce extra torque ripple in the SR motor.

B. case2

Similar results at rated speed $(1500 \mathrm{rpm})$ and rated load are shown in Figs. 14, 15, 16 and 17. At this operating point, the current (torque) is controlled by the angle control mode $[5,6]$. The $\mathrm{T}$-on and $\mathrm{T}$-off angles are fixed at $-3.75^{\circ}$ and $22.5^{\circ}$ respectively, dc excitation voltage is kept constant at 280 volt; speed of the motor is set at $1500 \mathrm{rpm}$ and the simulation is conducted under these conditions. The current, net flux and mutual flux-linkage in all the phases are plotted in Figs 14(i), 14 (ii) and 14 (iii). The results show that the current waveforms in all the phases are not the same. The peak current in $\mathrm{Ph} 1$ is maximum and that in $\mathrm{Ph} 2, \mathrm{Ph} 3$ and $\mathrm{Ph} 4$ are almost equal. At the commutation points, the current in $\mathrm{Ph} 4$ is maximum followed by $\mathrm{Ph} 1$. At this point the currents in $\mathrm{Ph} 2$ and $\mathrm{Ph} 3$ are the same but less than that in $\mathrm{Ph} 1$. The net fluxes in various phases (c.f. Fig. 14 (ii)) do not differ appreciably. Fig. 15 illustrates the difference between the self flux-linkage and the net flux-linkage of a particular phase. The energy conversion loops with and without the mutual fluxes are shown in Figs 16(i), 16 (ii), 16 (iii). The same results with mutual fluxes are compared for different phases in Fig. 17. These results show that the shape of the energy conversion loops are same for $\mathrm{Ph} 2$ and $\mathrm{Ph} 3$ but differ considerably for $\mathrm{Ph} 1$ and $\mathrm{Ph} 4$. Hence, the torque produced in
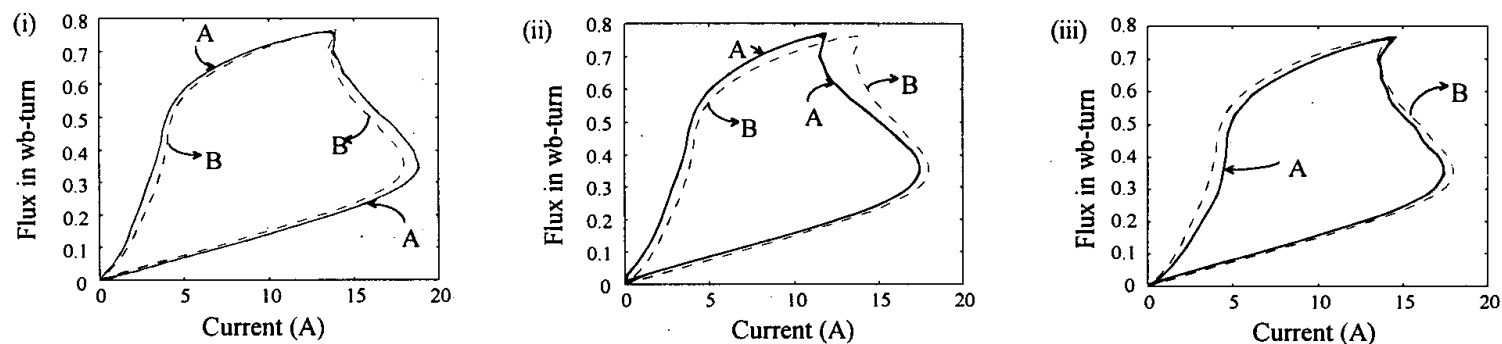

Fig. 16 Comparison of energy conversion loops (A) with mutual flux (bold line) and (B) without mutual flux (dotted) for (i) Ph1; (ii) Ph2 and Ph3; and (iii) $\mathrm{Ph} 4$ at $1500 \mathrm{rpm}$ and full load. 


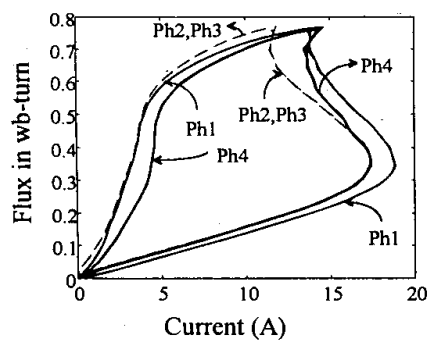

Fig. 17 Comparison of energy conversion loops (with mutual flux) of different phases at $1500 \mathrm{rpm}$ and full load

different phases will not be the same. Consequently, the windings in different phases will not be utilised equally.

\section{Discussion}

In order to achieve the same average torque from all the four phases at high speed, the T-on and T-off angles should not be the same for all the phases. Similarly at low speeds, and at a particular load, the reference currents should not be the same for all phases. Hence, for both low speed and high speed ,the current carrying capability of the different phases need not be the same for all the phases and the design of the windings can be made accordingly. For efficient utilisation of the machine this analysis can be made use of in the design stage.

(i)

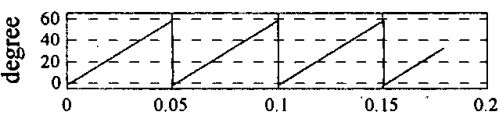

(ii)

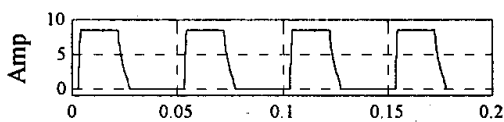

iii)

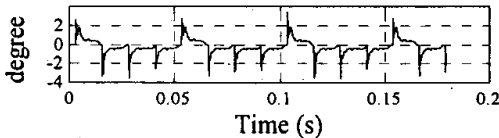

\section{Position Estimation}

Flux-current method $[7,8]$ is one of the most popular method for position estimation of SR motor. Measuring the voltage across and current through the winding, the flux-linkage of any phase may be computed using (2). Knowing the current and flux, the position of the rotor may be estimated using the off-line measured static flux-linklage characteristics. Lyon [7] suggested this method in early '90s. The off-line measured characteristics are solely based on self-flux linkage and phase current. They do not take into account the dynamic non-idelaities or mutual fluxes. In dynamic condition, flux value obtained through (2) is influenced by the mutual fluxes. Hence, the accuracy of the estimated position, using this dynamic flux and without correcting it for mutual flux, will be poor. In this paper, the off-line measured mutual flux is used for correcting the flux-linkage of any phase and this corrected flux is used for position estimation.

The effect of position estimation is studied first in simulation. The simulation results of estimated position, phase current and error due to mutual flux at $200 \mathrm{rpm}$ and $1400 \mathrm{rpm}$ are given in Fig. 18a and Fig 19a respectively. It shows that mutual fluxes alone can introduce an error of $\pm 3^{\circ}$. The same is verified in experiment. The experimental results are shown in Fig. $18 \mathrm{~b}$ and $19 \mathrm{~b}$ for the above operating conditions.

(i)

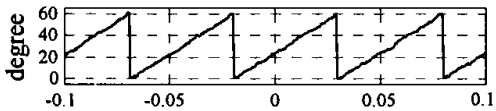

(ii)

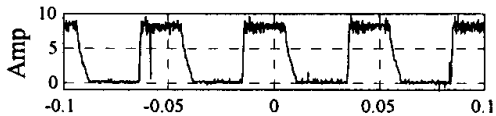

(iii)

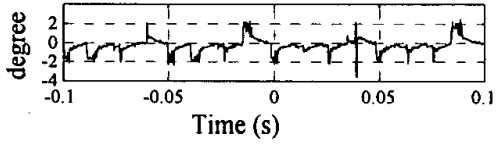

Fig. 18 (a) Simulation and (b) Experimental results of (i) Estimated position, (ii) Phasel current and (iii) Error in estimation due to mutual flux only, at $200 \mathrm{rpm} .\left(\mathrm{Vdc}=130\right.$ volt, $\mathrm{T}$-on $=3.75^{\circ}, \mathrm{T}$-off $=26.25^{\circ}$, load $\left.=11.5 \mathrm{Nm}\right)$

(i)

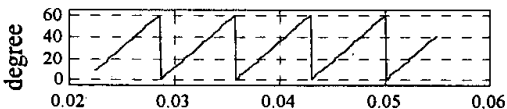

(ii)

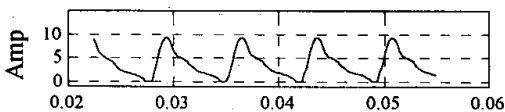

(iii)

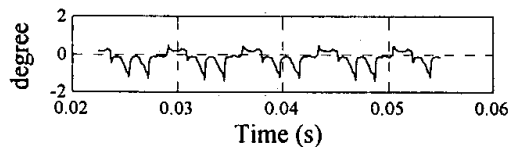

(i)

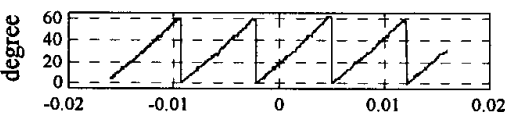

(ii)

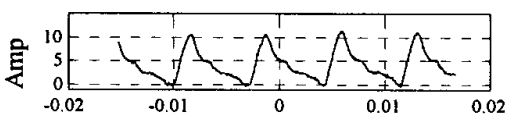

(iii)

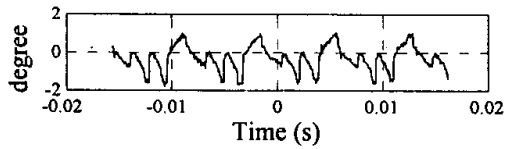

Fig. 19 (a) Simulation and (b) Experimental results of (i) Estimated position, (ii) Phasel current and (iii) Error in estimation due to mutual flux only, at $1400 \mathrm{rpm} .\left(\mathrm{Vdc}=130\right.$ volt, $\mathrm{T}$-on $=-5^{\circ}, \mathrm{T}$-off $=22.5^{\circ}$, load $\left.=7.5 \mathrm{Nm}\right)$ 
(i)

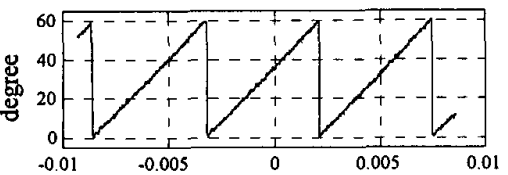

(ii)

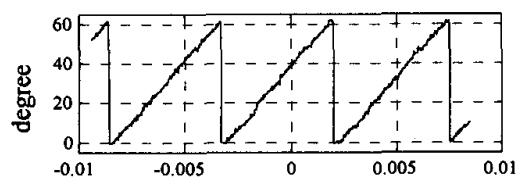

(iii)

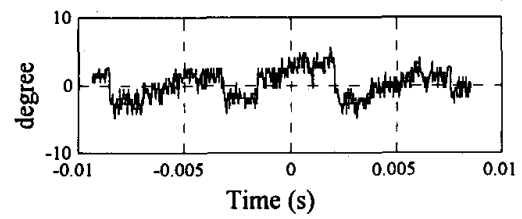

Fig. 20a Experimental result of (i) Actual Position, (ii) Estimated position and (iii) Net error at $1875 \mathrm{rpm}$ without mutual flux.

It is also observed the net error in position estimation is reduced after correcting for the mutual fluxes. A typical test result (at $1875 \mathrm{rpm}$ ) of estimated position with and without mutual flux, and actual position along with net error (actual-estimated) in estimation are given in Fig. 20a and 20b.

The above algorithm is implemented using a DSP platform (TMS320c50) and it is tested on a 4-phase, 8/6 pole, 4-kW OULTON motor. An IGBT based split-capacitor converter is used. The maximum switching frequency of the converter is limited to $10 \mathrm{kHz}$.

\section{v. CONCLUSION}

In this paper, the measurement of mutual flux between the adjacent phases are discussed and the test results are presented. The results show that the magnitude of mutual coupling in all phases are same but the direction is not same for all phases. The effects of mutual flux on phase current, phase flux and energy conversion contour for the different phases are demonstrated through simulation results. It is shown that for same $\mathrm{T}$-on, T-off, $\mathrm{I}^{*}$ and excitation voltage, the current and flux waveforms in different phases are unequal due to mutual flux. In effect the load sharing among the different phases is not same. For example the energy conversion due to $\mathrm{Ph} 4$ is considerably less than due to $\mathrm{Phl}$ (se Fig. 13(i) and Fig. 17). This will cause the under-utilisation of $\mathrm{Ph} 4$ compared to $\mathrm{Ph} 1$. Hence, for uniform utilisation of the different phases, the $\mathrm{T}$-on and $\mathrm{T}$-off angles for high speeds and current references for low speeds are suggested to be different. These findings may be used in the design stage as well.

A more accurate position estimation algorithm using the correction for mutual fluxes is demonstrated. The mutual flux, if not corrected, may introduce an error as much as $\pm 3^{\circ}$ in the estimated rotor position. (i)

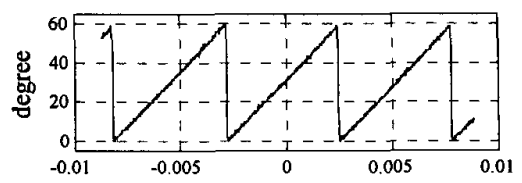

(ii)

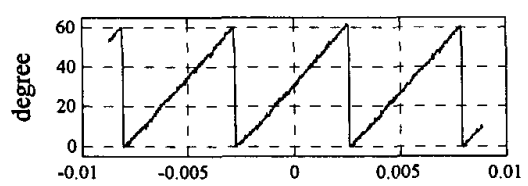

(iii)

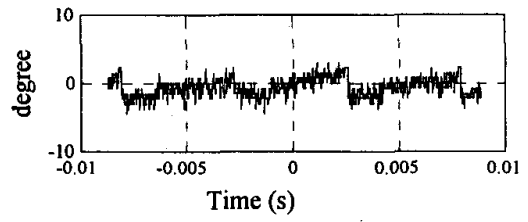

Fig. 20b Experimental results of (i) Actual Position, (ii) Estimated position and (iii) Net Error at $1875 \mathrm{rpm}$ with mutual flux.

\section{REFERENCES}

[1] Stephenson J. M., and Corda J., "Computation of torque and current in doubly-salient reluctance motors from nonlinear magnetisation data", Proc. IEE, 1979, 126, (5), pp. 393-396.

[2] Fulton N. N., and Stephenson J. M., "A review of switched reluctance machine design", International Conference on Electrical Machines, 1988.

[3] H.H.Moghbelli, G.E.Adams and R. G. Hoft, "Prediction of the instantaneous and Steady state torque of the switched reluctance motor using finite element method (FEM)", Proceeding of IAS annual meeting 1988, pp 59-70.

[4] V. Ramanarayanan, L.Venkatesha and D. Panda, "Flux-linkage Characteristics of Switched Reluctance Motor", International Conference on Power Electronics, Drives and Energy Systems for Industrial Growth (PEDES'96), Delhi, December 1996, pp 281-285.

[5] Miller T.J.E.,"Switched Reluctance Motors and Their Control", Magna Physics Publishing And Clarendon Press Oxford 1993.

[6] T.J.E. Miller, M. McGilp, "Nonlinear theory of the switched reluctance motor for rapid computer-aided design", IEE Proceedings, Vol. 137, Pt. B, No.6, November 1990, pp 337-346.

[7] J. P. Lyons, S. R. Macmin, M. A. Preston, "Flux/Current Method for SRM rotor position estimation", IEEE-Industry Application Society Annual Meeting, 1991, Vol.1, pp. 482-487.

[8] Debiprasad Panda, V. Ramanarayanan, "An Accurate Position Estimation Method for Switched Reluctance Motor Drive", PEDES '98, Perth, Australia, December, 1998, pp 523-528. 